\title{
Segmentation-Based Video Coding System Allowing the Manipulation of Objects
}

\author{
Philippe Salembier, Ferran Marqués, Montse Pardàs, Josep Ramon Morros, Isabelle Corset, \\ Sylvie Jeannin, Lionel Bouchard, Fernand Meyer, and Beatriz Marcotegui
}

\begin{abstract}
This paper presents a generic video coding algorithm allowing the content-based manipulation of objects. This manipulation is possible thanks to the definition of a spatiotemporal segmentation of the sequences. The coding strategy relies on a joint optimization in the rate-distortion sense of the partition definition and of the coding techniques to be used within each region. This optimization creates the link between the analysis and synthesis parts of the coder. The analysis defines the time evolution of the partition, as well as the elimination or the appearance of regions that are homogeneous either spatially or in motion. The coding of the texture as well as of the partition relies on region-based motion compensation techniques. The algorithm offers a good compromise between the ability to track and manipulate objects and the coding efficiency.
\end{abstract}

Index Terms - Affine motion estimation, compensation, ratedistortion optimization, region-based coding, spatiotemporal segmentation, video coding.

\section{INTRODUCTION}

$\mathbf{C}$ ONTENT-BASED representation and coding of the visual information is currently becoming an extremely active field of research. An important part of this research is stimulated by some of the MPEG-4 activities that try to provide technical solutions to emerging needs of applications such as interactive video services, video mobile terminals, remote control, etc. In a large number of these applications, there is a need to have a content-based representation of the sequence instead of a more traditional pixel-based or blockbased representation. One way of facing this problem is to rely on signal-dependent partitions of the sequence where regions ideally define the various objects of the scene. Then, all processing steps, including representation, processing, coding, or manipulation, rely on these regions.

Defining a content-based representation of sequences by means of partitions has at least three consequences.

- The signal-dependent partition results from an analysis of the sequence. In particular, a priori defined partitions such as block partitions are not suitable.

Manuscript received March 10, 1996; revised July 1, 1996. This paper was recommended by Guest Editors Y.-Q. Zhang, F. Pereira, T. Sikora, and C. Reader. This work has been supported by the European Community through the RACE MAVT and MORPHECO projects.

P. Salembier, F. Marqués, M. Pardàs, and J. R. Morros are with the Polytechnic University of Catalonia, 08034 Barcelona, Spain.

I. Corset, S. Jeannin, and L. Bouchard are with Philips Research Laboratory, Limeil-Brévannes 94453, France.

F. Meyer and B. Marcotegui are with the Paris School of Mines, 77305 Fontainebleau, France.

Publisher Item Identifier S 1051-8215(97)00884-7.
- The representation of objects by partitions does not only involve the definition of the objects' contours at one time instant but also their time evolution. Indeed, region or object tracking is mandatory for a large number of content-based functionalities [18]. This requirement eliminates all techniques that define partitions independently from one frame to another.

- The representation cannot rely on a fixed topology of the partition. Indeed, the partition has to evolve with the modifications of the scene content: regions are to be introduced or removed in the partition when new objects appear or disappear in the scene.

Coding algorithms following this strategy can be viewed as second generation coding approaches [11]. Examples of region-based video coding schemes can be found in [19], [37], [4], [15], [9], [3], and [32]. The main difference between these techniques is the relative importance they assign to the spatial or the motion information. In a first set of examples [37], [15], [32], regions are characterized by their spatial homogeneity, whereas in a second set [19], [4], motion information is used as the main homogeneity criterion. Finally, examples of segmentation techniques involving both criteria can be found in [9] and [3]. This last approach offers a large number of advantages: the spatial homogeneity criterion allows a very accurate definition of the regions and the processing of generic sequences because, for example, it can deal with the appearance of new objects. The motion homogeneity criterion is important to limit the number of regions and therefore the coding cost associated with the partition. This limitation is generally obtained by merging spatial regions that can be processed, that is compensated, together.

All these techniques have the same structure involving an analysis phase (mainly the segmentation) followed by a synthesis step (mainly the coding functions). In most of them, there is no strong relation between the two steps. One tries to obtain the "best" segmentation, on the one hand, and then, to code the partition as well as the texture with the lowest possible amount of bits, on the other hand. However, high coding efficiency requires a strong interaction between the analysis and synthesis steps. Indeed, the definition of the partition should depend on the techniques used to code the various regions. Based on the work done for bit allocation reported in [34], [20], and [25], a solution for an optimal definition of a partition and of the set of coding techniques to be used in each region is proposed in [26]. However, in this work, the partitions are defined for each frame independently 
of the previous partitions. Therefore, there is no time tracking possibility of the regions and the scheme is not really suitable for an efficient manipulation of objects.

This paper describes a video coding system that combines the advantages of the approaches mentioned above in order to allow an efficient manipulation of objects. The main features of the proposed algorithm are the following: 1) the segmentation involves both spatial and motion homogeneity criteria, 2) the time evolution of each region is defined (time tracking), and 3) the definition of the coding strategy involves a global optimization of the partition as well as of the coding technique for each region. Moreover, the scheme is quite flexible and open to the integration and comparison of new tools. A first version of the algorithm has been proposed within the framework of MPEG-4 under the name of SESAME. A detailed description of the algorithm and of its hardware complexity can be found in [6].

The organization of this paper is as follows: Section II describes the encoding algorithm and gives some details on the main processing steps. Section III is devoted to coding results and discusses the possible use of the algorithm for building new content-based functionalities. Finally, conclusions and open issues are reported in Section IV.

\section{CODING Algorithm}

\section{A. Structure of the Algorithm}

The objective of this section is to give an overview of the algorithm and to describe the basic coding strategy. The block diagram corresponding to the encoding process is presented in Fig. 1. The encoding process relies on three sets of functions: bit allocation function, partition functions, and coding functions.

Bit Allocation Function: This function, corresponding to the Decision block of Fig. 1, makes the link between the analysis (partition functions) and the synthesis (coding functions) parts of the encoder. As discussed in the introduction, an efficient content-based representation relies on a careful study of the bit allocation problem. The objective is to share a given number of bits between the various types of information to be coded and transmitted (motion, partition, gray level, and color). As a result, the Decision block defines the coding strategy, that is the set of regions to be coded as well as the type of coding technique to be used in each region. The coding strategy results from the optimum (in the rate-distortion sense) selection of regions and coding techniques out of a set of possible regions and coding techniques.

Partition Functions: The objective of this set of functions is to create for each frame a universe of possible regions out of which the Decision has to create the final partition. Note that, to be able to track objects, this universe of regions should be related to the previous partition. This is the objective of the Projection block of Fig. 1. Moreover, the universe of regions has to offer to the Decision the possibility to introduce new regions or to eliminate past

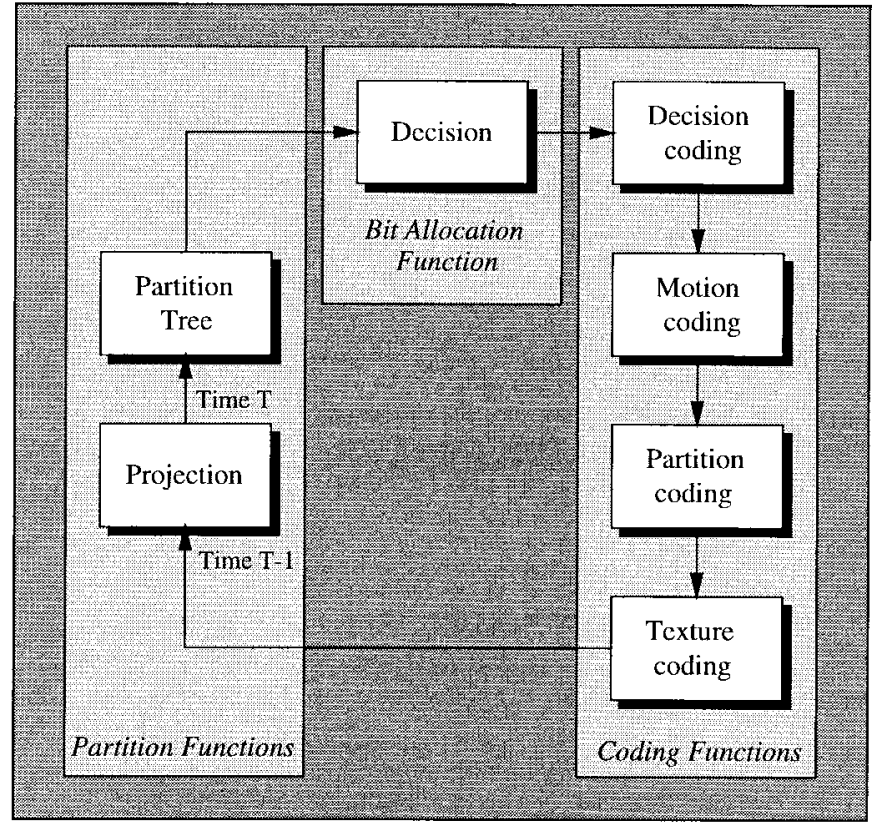

Fig. 1. Description of the encoding processing steps.

regions. This function is achieved by the Partition Tree block.

Coding Functions: The last set of functions actually codes the information necessary to restore the sequence on the receiver side. It deals with the encoding of the coding strategy (Decision coding), the motion information (Motion coding), the partition (Partition coding), and the pixel values (Gray level and color) called Texture in the sequel. To have an efficient representation, both the partition and the texture are motion compensated. This explains why the Motion coding block is located before the Partition and Texture coding blocks.

In the sequel, the various processing steps are further explained and discussed.

Projection Block: The objective of this block is to define the time evolution of the regions. To limit as much as possible the processing delay and the computational load, only the previous coded frame and its partition at time $T-1$ are used to define the time evolution of the partition at time $T$. This step is purely a region tracking step [22] and, in particular, new regions cannot be introduced. Note, however, that some regions may disappear. The projection block will be more precisely described in Section II-B.

Partition Tree Block: The main objective of this block is to create the universe of regions out of which the Decision has to create the final partition. Assume, in a first step, a situation where the scene content is not strongly modified from one frame to the next one. In this case, the partition as defined by the Projection, called the projected partition in the sequel, is a rather good approximation of the optimal partition of the current frame. However, some new objects may have appeared in the scene and new regions may need to be introduced. On the other hand, several regions belonging to the same object 


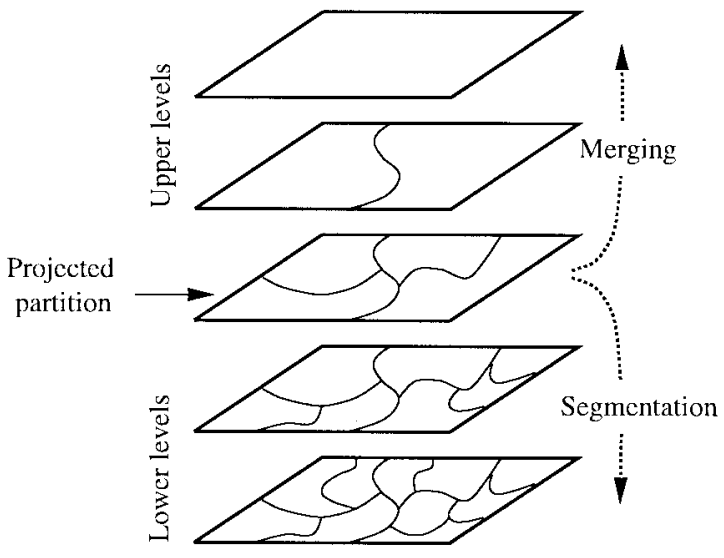

Fig. 2. Structure of the partition tree.

can be processed globally because, for example, they have the same motion. In this case, these regions can be merged to create one single object and to decrease the coding cost devoted to the partition. In conclusion, some fluctuations with respect to the projected partition must be allowed. The concept of partition tree [24] can be used to deal with these fluctuations.

As illustrated in Fig. 2, the partition tree is formed by the projected partition plus a set of hierarchical partitions that are "above" and "below" the projected partition. Below the projected partition, several levels of finer partitions are created. The partitions are finer in the sense that they involve a larger number of regions and that the contours present at a given level are also present at lower levels. This procedure can be viewed as a hierarchical segmentation that splits the regions of a given level to produce the regions of the next lower level [27]. Note that the procedure is purely spatial: it does not take into account any motion information. Above the projected partition, several levels of coarser partitions are created. Here, regions are merged if they can be processed globally: this reduces the partition coding cost and goes closer to the notion of objects. In practice, following a motion homogeneity criterion, regions are successively merged to create coarser partitions. In Section III, we will see how this initial merging strategy can be modified to deal with specific content-based functionalities.

As can be seen, the partition tree defines the universe of possible regions issued from the projected partition. These regions can be homogeneous either spatially (lower levels of the tree) or in motion (upper levels of the tree). No decision is made concerning the actual partition to be coded. The objective is simply to define a reduced set of regions that are likely to be part of the optimum partition.

Finally, let us mention that, besides the definition of the universe of regions, the partition tree block also includes the estimation of the motion parameters for all regions in the tree. This estimation is necessary for the creation of the upper levels of the tree and also for the Decision. Section II-C discusses more precisely the creation of this tree.

Decision Block: As previously discussed, based on the ratedistortion criterion, the Decision block selects the best strategy in terms of regions and coding techniques among a set of possibilities [26], [17]. The partition tree contains all regions that may belong to the final partition; for each of them, a set of possible coding techniques is proposed to the Decision. This last set involves several region-based coding techniques with various levels of quality. Moreover, the techniques can be proposed in intraframe mode (coding of the original signal) and in interframe mode (motion compensation of the region and coding of the prediction error).

Fig. 3 summarizes the decision process: from the partition tree, all regions are extracted to form the decision tree. Several region coding techniques (defined by the set of coding techniques $\{C 1, \cdots, C n\}$ ) are considered for each region. Then, taking regions from various levels of the partition tree, the Decision block defines jointly the best partition and the best coding technique for each region. More details about this block can be found in Section II-D.

Coding Blocks: Once the optimum partition and the coding strategy have been chosen, the information necessary to decode the sequence is sent to the receiver. This information is composed of the coding strategy itself, the motion parameters for the regions that should be compensated, the partition, and finally, the texture parameters of each region. A large set of coding techniques can be used. Sections II-E and II-F describe more precisely the various techniques that have actually been used.

Intraframe mode of transmission is necessary to initiate the coding process and to periodically refresh the information at the receiver side. The description previously given assumed an interframe mode of coding. For the intraframe mode, two modifications have to be introduced. First, the Projection does not rely on the previously coded partition. Therefore, this step is replaced by a simple initial segmentation of the frame. Second, the Decision process optimizes the coding strategy on the basis of the partition tree and of only intraframe coding techniques.

\section{B. Projection}

The partition projection adapts the partition $P_{T-1}$ of frame $T-1$ to the current frame [32]. $P_{T-1}$ is the partition chosen by the decision for representing the previous image. The projection procedure accommodates $P_{T-1}$ to the data of image $T$, without introducing new regions.

In the projection algorithm, the region correspondence problem can be solved using connectivity and motion criteria [22], [21]. The projection is performed in two steps. First, motion is estimated between the original frames $T-1$ and $T$, and the previous partition is motion compensated. Then, the definition of regions into the current frame is achieved by a three-dimensional (3-D) watershed algorithm [16] which is essentially a morphological region growing algorithm that extends the compensated past regions (called markers) into the current frame.

In the work presented in [21], the compensated regions used as markers and extended by the watershed algorithm are assumed to be spatially homogeneous. This assumption cannot be made now because partitions are formed by regions that may be homogeneous either in gray level or in motion. 


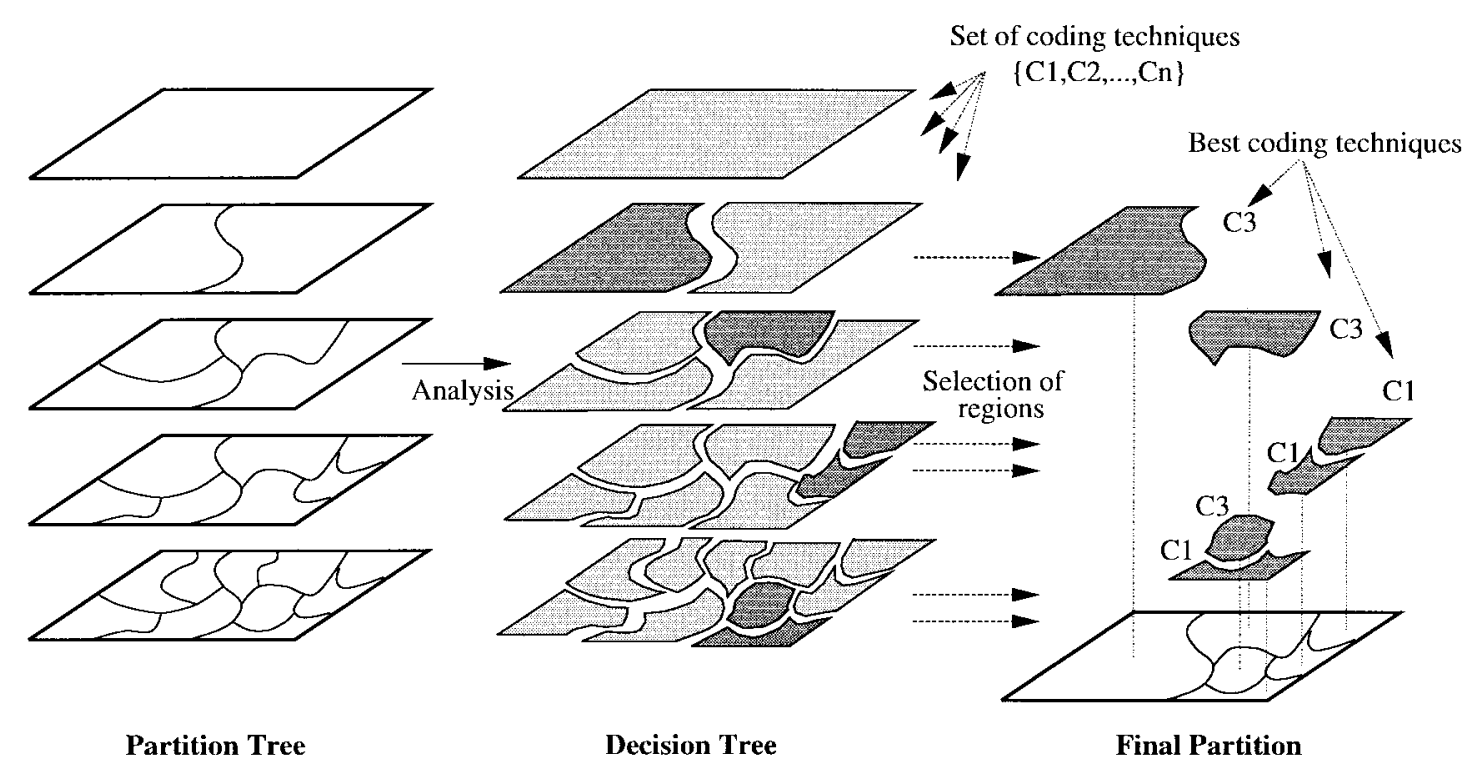

Fig. 3. Decision process.

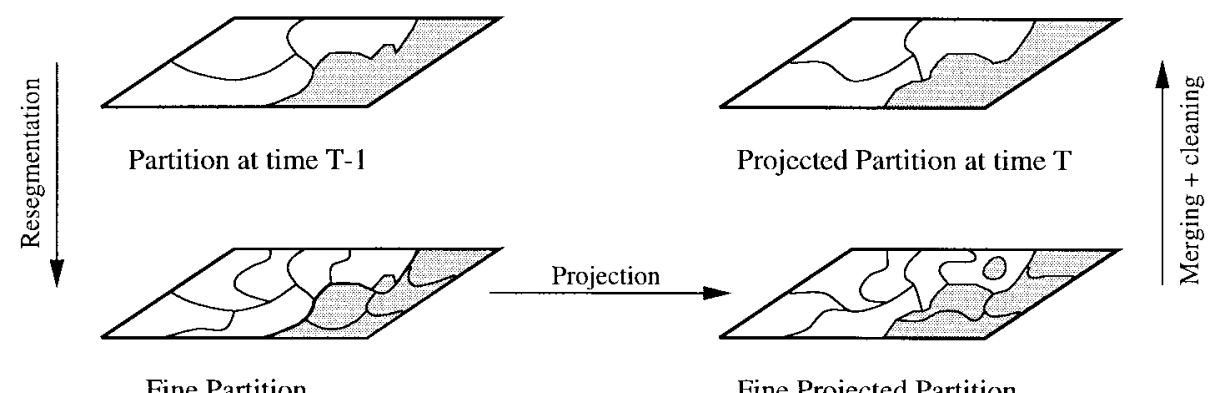

Fine Partition

Fine Projected Partition

Fig. 4. Example of projection.

To solve this problem, the Projection block uses a double partition approach [13]. In this approach, two different levels of partition are defined. The partition of the previous image $P_{T-1}$ is resegmented in order to achieve a finer partition as shown in Fig. 4. This fine partition contains a larger number of regions which are obtained by resegmenting the regions in $P_{T-1}$ following spatial criteria. The objective is to guarantee the spatial homogeneity of each region.

This fine segmentation is then projected in the current frame in order to obtain a fine segmentation at time $T$. As mentioned previously, a 3-D watershed algorithm is used for this fine partition projection [16], [21]. The algorithm can be seen as a region growing technique that progressively assigns pixels $p_{i}$ to regions $R_{j}$. The assignation order is defined by a cost function assessing the "distance" between pixel $p_{i}$ and region $R_{j}$. The cost of assigning a pixel $p_{i}$ to a region $R_{j}$ uses three different types of information

$$
\begin{aligned}
\operatorname{Cost}\left(p_{i}, R_{j}\right)= & \alpha_{1} \operatorname{dist}_{t}\left(p_{i}, R_{j}\right)+\alpha_{2} \operatorname{dist}_{c}\left(p_{i}, R_{j}\right) \\
& +\alpha_{3} \operatorname{dist}_{d}\left(p_{i}, R_{j}\right) .
\end{aligned}
$$

The three functions dist ${ }_{t}$, dist ${ }_{c}$, and dist ${ }_{d}$ are the distances related to the texture, contour complexity, and deformation information, respectively. The function dist $_{t}$ computes the difference between the gray level value of a pixel $p_{i}$ with respect to the mean value of a region $R_{j}$. In turn, the function dist $_{c}$ is related to the increase in contour complexity of a region $R_{j}$ if a pixel $p_{i}$ is added to it. Finally, the function dist $_{d}$ measures the deformation of a region $R_{j}$ with respect to the projected marker when adding a pixel $p_{i}$ to it. The use of this cost function provides a good stability of the labels through the time domain, and, therefore, allows an efficient tracking of the objects [12].

In order to obtain the partition $P_{T}$, only those contours of the fine projected partition associated to the coarse partition have to be kept. This can be easily done since the projection algorithm keeps track of the labels of each region. Therefore, the projection of a region from the coarse partition can be obtained by merging the projections of the regions that belong to it at time $T$; that is, by relabeling the fine projected partition. However, the projection and relabeling of the fine partition does not ensure that an actual partition is obtained. Indeed, unconnected regions may have the same label after relabeling. This problem is solved by a cleaning step that keeps the largest connected component for each label and removes the other connected components with the same label. A pure twodimensional (2-D) watershed algorithm is applied to obtain the 
final partition. The complete procedure is illustrated in Fig. 4, where the evolution of a region is shown.

\section{Partition Tree and Motion Estimation}

1) Creation of the Partition Tree: The objective of the partition tree is to define a universe of regions for the Decision. To limit the complexity of the Decision, this universe should be of reasonable size and structured in a hierarchical way [24]. This goal can be achieved by creating fluctuations around the projected partition: lower levels are generated by successive hierarchical segmentation steps [27], [32], and upper levels are obtained by successive merging.

a) Merging procedure: On the upper levels of the partition tree, coarser partitions are created by merging steps following a motion criterion. The aim is to merge neighboring regions that have a similar motion. Indeed, if these regions can be compensated using the same motion parameters, the contour between them does not need to be coded and only one set of motion parameters is needed. The merging procedure is iterative: starting from the projected partition, a first coarser partition is created. Then, starting from this partition, a new coarser partition is created. This process is repeated up to the highest level of the partition tree.

At each level, before the merging itself, the motion of each region is estimated. This procedure will be detailed in Section II-C2. It results in a set of six parameters defining an affine transformation for each region. Then, a region adjacency graph (RAG) is constructed. Each node of the RAG represents a region and the edges of the graph indicate the neighboring relationship between regions.

The edges of the RAG are valued by assigning to them a merging cost that estimates the motion difference of two regions. The cost that has been used is the increase of mean square error (MSE) in the compensation of the union of the two regions (with the set of motion parameters of the region that gives the lowest MSE) with respect to the MSE obtained when the two regions are compensated separately. Finally, a given number of merging steps are performed by selecting in the RAG the required number of edges with lower cost.

b) Resegmentation procedure: The lower levels of the partition tree are created by resegmenting the regions of the projected partition. This segmentation creates new regions that can represent new objects appearing in the scene, or objects with several components that have been previously merged together but are starting to undergo different motions. In both cases, we need to separate regions with a spatial criterion.

As the merging, the segmentation is iterative and progressively builds the lower levels of the partition tree by performing several hierarchical segmentations. Note that the problem is similar to the one of creating the fine partition in the projection step, but the procedure has to be done now in a hierarchical way. The morphological approach described in [27], [30], and [32] is particularly suitable for this step: without getting into details let us mention that this segmentation approach involves 1) the computation of the difference between the original frame and the mean of each region called "residue," 2) the simplification of the residue by "connected operators" [31], 3) a marker extraction step, and 4) finally a watershed algorithm [16]. In our implementation, all segmentation steps are size-oriented except the last one which is contrast-oriented (see [27], [30], [32], and [24] for more details). One of the advantages of this segmentation procedure is that it gives good results at a low computational cost.

2) Motion Estimation: As mentioned previously, the motion of each region of the partition tree has to be estimated for the merging steps and for the Decision.

a) Motion representation: The motion of each region is represented by a polynomial model. A simple translational model is generally not sufficient for regions of medium or large size. Parametric motion models [7], [36] are able to give a region-based motion representation that is both compact and able to deal with quite complex motions.

In practice, on each region $X$ of the partition tree, the motion between previous $(T-1)$ and current $(T)$ frames is assumed to be affine, i.e., defined by two polynomials of order one

$$
d x=a+b x+c y, \quad d y=d+e x+f y
$$

where $(d x, d y)$ is the motion of pixel $(x, y)$ between $T-1$ and $T$. This model can handle translations and simple rotations of any planar facet. Of course, to avoid expanding the bit rate by using models that are more complex than needed on some regions (either very small or with simple motion), the model complexity is adapted to the region it characterizes.

b) Estimation process: The estimation of the model parameter is done assuming constancy of the luminance along the regions' trajectories [36]

$$
I(x, y, T)=I(x-d x, y-d y, T-1)
$$

where $I(x, y, T)$ denotes the intensity value at location $(x, y)$ at time $T$.

The estimation itself is done by differential methods [33]. The objective function is the MSE between the original region and its current prediction. The Gauss-Newton method is used as minimization algorithm. This method iteratively minimizes the region MSE by following the direction of its gradient that has been locally linearized. This approach represents a good tradeoff between complexity and reliability. After the final step of the minimization, a spatial relaxation is performed. It relies on a locally controlled propagation of the current motions parameters to neighbor regions.

c) Estimation framework: It is well known that gradientbased minimization methods do not insure the convergence toward the absolute minimum. The situation is even worse here because regions are of arbitrary size and the sharp gray level transitions, which are of great help for the convergence, correspond most of the time to the borders of the regions and are therefore difficult to exploit. To improve the behavior of the algorithm, the minimization process is carefully initialized and, furthermore, embedded in a multiresolution framework.

For each region, the initialization tests several sets of motion parameters and selects the one that produces the lowest MSE. In particular, the set of parameters estimated for this region in the previous frame (this is possible thanks to the time 


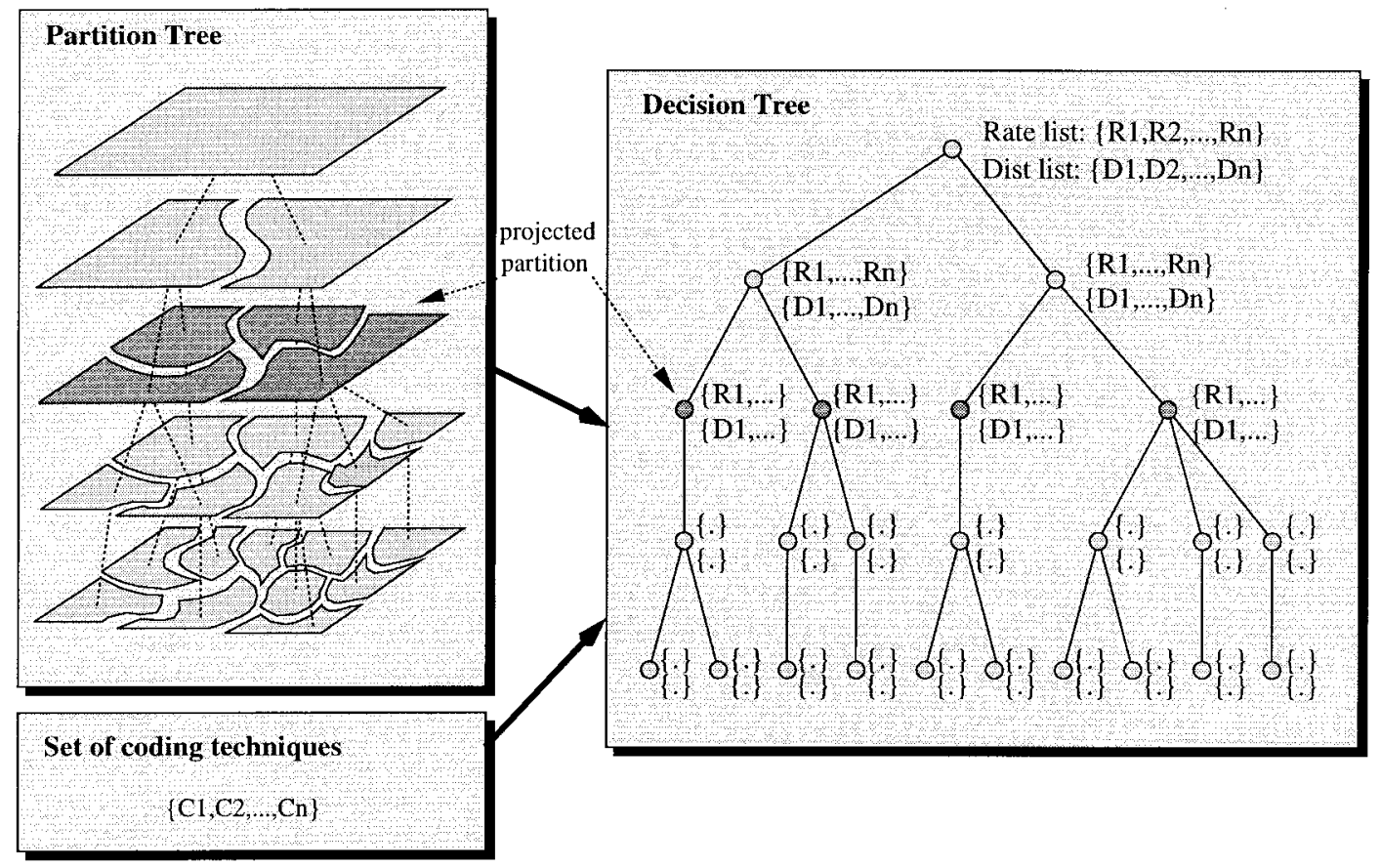

Fig. 5. Construction of the decision tree.

tracking ability of the system), as well as the set of parameters of regions located at the same position but on other levels of the partition tree are tested. Finally, the estimation is done following a multiscale coarse-to-fine strategy based on a Gaussian pyramid.

\section{Decision}

The goal of the Decision step is to select the best coding strategy among a set of possibilities that are represented by the partition tree and a list of coding techniques. The partition tree defines the set of regions out of which the algorithm should create the final partition. The list of coding techniques defines not only the type of techniques, but also their parameters (for example quantization steps) and the intraframe or interframe mode choice. To limit the processing delay, the strategy is optimized for each frame separately. The Decision process relies on the concept of Decision Tree [26], [17] illustrated on Fig. 5. This tree concentrates in a compact and hierarchical structure all the possible coding choices. The partition tree defines the choices in terms of regions. The list of coding techniques deals with the actual coding of these regions.

The structure of the decision tree is defined by the partition tree: each node of the decision tree corresponds to a region in the partition tree. The relations Father/Children between the nodes are also given by the partition tree and define how one region at a given level may either be split in various regions (Children) or be merged to form a larger region (Father). To define the coding strategy in the rate-distortion sense, the decision tree should also convey the information about the coding cost (rate measured in number of bits) and quality (distortion assessed by the squared error) of all possible coding techniques. Therefore, a list of rates ("Rate list" in Fig. 5) and a list of distortion ("Dist list" in Fig. 5) are assigned to each node. In practice, each region of the partition tree is coded by all techniques (with various quality levels and either in intraframe mode or in interframe mode) and the corresponding rate and distortion values are stored in the decision tree. The computation of the distortion is rather straightforward. We have used the squared error between the original and coded frames (that is, the sum of squared difference between the values of the original and coded frames for all pixel belonging to the region support). The situation is more complex, however, for the computation of the rate. Indeed, the rate associated with a region is composed of the sum of the number of bits devoted to the texture, the motion, as well as the shape information. In practice, the texture and the motion information are coded independently for each region so there is no major difficulty in defining the texture or the motion rate for each region. But this independence is not maintained for the shape information because a contour is always shared by two regions. In order to avoid the problem of optimization with complex dependency between regions, we have used the following approximation of the shape rate: based on the analysis of a large number of coded frames, an average number of bit per contour points has been computed and the shape rate assigned to a region is equal to this average figure multiplied by the region perimeter divided by two (each contour point is shared by two regions). Finally, note that this step of construction of the decision tree is simply a phase of evaluation of the respective merits of each technique and no decision is taken.

The optimization relies on the technique discussed in [20], [25], and [26]. The problem can be formulated as the minimization of the distortion $D$ of the image with the restriction 
that the total cost $R$ be below a given budget (defined for each frame). It has been shown that this problem can be reformulated as the minimization of the Lagrangian: $D+\lambda R$ where $\lambda$ is the so-called Lagrange parameter. Both problems have the same solution if we find $\lambda^{*}$ such that $R$ is equal (or very close) to the budget. Therefore, the problem consists in using the decision tree to find a set of regions creating a partition and a set of coding techniques minimizing $D+\lambda^{*} R$. Assume in a first step that the optimum $\lambda^{*}$ is known. How can the best set of regions and coding techniques be selected?

- The first step is to make a local analysis and to compute, for each node, the Lagrangian for each coding technique. The technique giving the minimum Lagrangian is considered as the optimum one for this node and this Lagrangian is stored.

- The second step is to define the best partition. This can be done by a bottom-up analysis of the decision tree. Starting from the lowest level, one checks if it is better to code the area represented by a set of children regions as a single region $X$ or as a set of individual regions $\left\{x_{i}\right\}_{i}$ with $X=\cup x_{i}$. The selection of the best choice is done by comparing the Lagrangian of $X$ with the sum of the Lagrangians of $x_{i}$. If the former is lower than the latter, the node corresponding to $X$ is activated and the children nodes are deactivated. This procedure is iterated up to the root node. Note that, in order to use this approach, the distortion should be additive over the regions. In our experiments, the squared error has been used; however, any additive measure can be used. Moreover, it should be noticed that the approach highly relies on the hierarchical structure of the regions in the partition tree.

At the end of the procedure, the best partition is defined by picking up all the regions corresponding to the activated nodes together with their corresponding best coding technique (defined during the first step of the algorithm).

The definition of the optimum $\lambda$ parameter can be done with a gradient search algorithm. The algorithm starts with one very high value $\lambda_{h}\left(10^{20}\right)$ and one very low value $\lambda_{l}(0)$ of $\lambda$. For each value of $\lambda$, the optimization procedure described above is performed. This results in two coding strategies (partition and coding techniques) that should give rates $R_{h}$ and $R_{l}$, respectively, below and above the budget. If none of these rates is close enough to the budget, a new Lagrange parameter is defined as $\lambda=\left(D_{h}-D_{l}\right) /\left(R_{l}-R_{h}\right)$. The procedure is iterated until the rate gets close enough to the budget.

In this formulation of the optimization problem, the main parameter is the budget that is assumed to be given for each frame. Based on this budget, the algorithm finds the coding strategy that minimizes the distortion. In practice, this procedure creates a coded sequence with a variable quality. The same structure can be used to define a coding strategy leading to constant quality sequences. The only modification consists in defining a target distortion value for each frame and in inverting the role of $D$ and $R$ in the previous explanation. In this last case, the decision minimizes the coding cost to reach a given distortion.

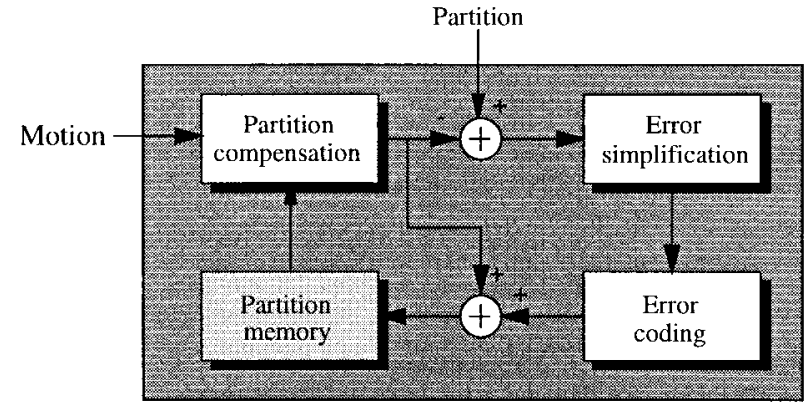

Fig. 6. Motion compensation loop for partition coding.

However, in practice, an intermediate solution may be used. Indeed, working at a fixed cost per frame may produce some frames of very poor quality (scene changes, complex motion). Most of the time, it is more efficient to spend more bits for these frames so that the quality is not too low and so that it may be possible to use these frames for the compensation of future frames. Therefore, the optimization works basically on a fixed nominal budget, but a minimum signal-to-noise ratio for each frame is defined. If this minimum signal-to-noise ratio is not reached with the nominal budget, the budget is progressively increased. The procedure is stopped when the decision has found the optimum strategy: 1) the distortion is minimal, 2) the budget is at least equal to the nominal budget, and 3) the signal-to-noise ratio is above a given threshold.

\section{E. Partition Compensation and Coding}

1) Compensation of Partitions: This section describes the coding of the partition sequence. The information to be transmitted to the receiver is made of three components: the shape (contour), the position, and the label of each region. To efficiently transmit this information, the basic coding strategy for partition coding relies on motion compensation [28], [29]. This approach is similar to the one classically used for texture motion compensation, but it is applied here on the partition information, that is on an image of labels. Therefore, as illustrated in Fig. 6, the partition coding involves the prediction by motion compensation, the computation of the prediction error, the simplification of the error, and the coding of the simplified error.

Before compensation, the motion parameters of the various regions of the current partition have been estimated in a backward mode. This step has been achieved in the partition tree block. The first problem to solve is to define what kind of information can be used to motion compensate the partition. Indeed, the motion of the pixels inside a region (texture motion) may not be equivalent to the motion of its shape. Both motions coincide in the case of a rigid foreground region. But, for instance, this is not the case for a background region because the modifications of its shape or of its contours are defined by the motion of the regions in its foreground. However, the texture motion can be used to compensate both the partition and the texture if extra information called the order is transmitted with the motion parameters [23].

The compensation itself can work either in a forward mode or in a backward mode. The second mode is generally more 


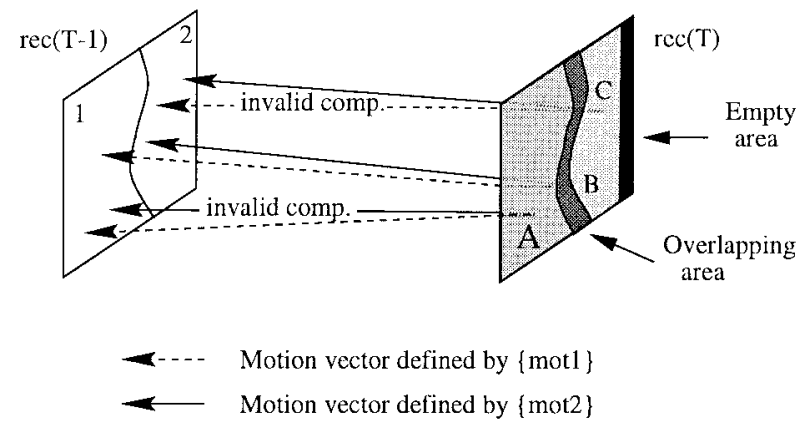

Fig. 7. Backward motion compensation of partition.

simple and is assumed in the following [28]. Let $\operatorname{seg}(T)$ and $\operatorname{rec}(T)$ denote the original (as defined by the Decision) and coded partitions at time $T$. In general, the partition coding is lossy, therefore, $\operatorname{rec}(T) \neq \operatorname{seg}(T)$. The backward mode of compensation is illustrated in Fig. 7. It is a backward mode in the sense that, for each pixel of $\operatorname{rec}(T)$, one tries to find its label in $\operatorname{rec}(T-1)$. In this case, the main problem is to define which motion vector has to be used when the pixel $(x, y)$ of $\operatorname{rec}(T)$ is considered. Indeed, at this stage, the receiver knows the set of motion parameters for each region, but not the current partition. Therefore, it does not know the region the pixel belongs to and it cannot select its corresponding motion parameters. The solution consists in considering at each pixel location all possible vectors defined by all possible regions. In the case of Fig. 7, there are two regions; therefore, two sets of motion parameters are considered for each point: one given by the parameters assigned to region $1\{\operatorname{mot} 1\}$ and one given by the parameters assigned to region $2\{\operatorname{mot} 2\}$. Each time a vector defined by the motion parameters of region $n$ does not point to a pixel belonging to region $n$ in $\operatorname{rec}(T-1)$, the compensation is invalid and discarded. In Fig. 7, this is the case for one of the two vectors used for points A and C. However, after each point has been considered, some pixels have no valid compensation (empty areas) and some others have more than one candidate (overlapping areas, see point B of Fig. 7). To solve the conflicts of overlapping areas, extra information called the order [23] is used. The order information defines which region is considered to be in the foreground of which region. In case of conflicts between labels, the foreground region gives the correct label. The reader is referred to [28] and [29] for the detailed description of order estimation algorithms. The problem of overlapping areas is in practice quite important when using affine motion models. However, the use of the texture motion and of the order is a quite efficient solution, because the texture motion information leads to a good compensation of the texture, and the order only represents a small amount of information necessary to compensate the partition. The order information is also useful for content-based representation implying multilayer representations. Finally, the empty areas are left without label and are processed as compensation errors.

2) Coding of the Partition: Once the order has been estimated, the coding of the partition can be done. The structure

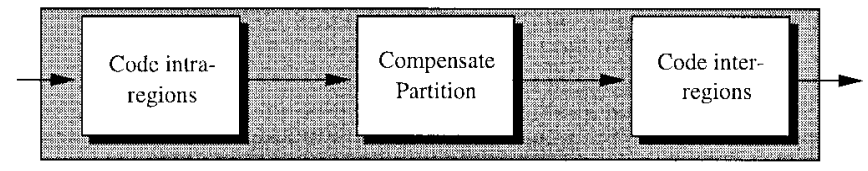

Fig. 8. Structure of the partition coding.

of the partition encoding process is illustrated by Fig. 8. First, all regions that have to be sent using the intraframe mode of the partition coding are processed. These regions are either regions that have no motion parameters because the Decision has defined an intraframe mode for texture coding or regions that produce very large shape errors if they are compensated. The second step consists in computing a partition involving both the regions sent in intraframe mode and the compensated regions. Finally, the partition errors are extracted, simplified, and coded. In the sequel, we describe briefly these three blocks (see [28] and [29] for more details).

a) Code intraregions: The objective of this block is twofold: first, to send the contour information of the regions transmitted in intraframe mode and second, to create a binary mask defining where the partition is considered as being already defined. During the compensation, each time a label is compensated at a location corresponding to a region transmitted in intraframe mode, the compensated label is not taken into account.

For the coding itself, almost all partition coding techniques (lossy or lossless) may be used (see [29]). In our implementation, the modified chain code described in [14] has been used. This coding step allows the restoration on the receiver side of the contours of the intraregions. However, it does not say which regions are transmitted in intraframe mode. To actually create the binary mask at the receiver side, a binary code is sent for each region.

b) Compensate partition: The compensation of the previous partition is done as described in Fig. 7. In case of conflict between several labels, a decision is taken on the basis of the order information. At the end, the compensated information is postprocessed in order to create a partition where each region is made of only one connected component (cleaning step).

c) Code interregions: The partition encoding in interframe mode consists in computing the error of the compensated partition, in simplifying this error, and in sending the information about the contours and labels of this error.

1) Compute error partition: The error computation consists in extracting the pixel locations where the compensated label is different from the label of the partition defined by the Decision. This results in an error mask.

2) Simplify error partition: This step introduces the losses in the coding process. Each region of the error mask is examined to know whether it has to be preserved and coded or discarded. In practice, two criteria can be used:

a) geometrical criterion: an error region is discarded if its size is smaller than a given size;

b) gray level criterion: an error region is discarded if its use in the coding does not introduce a strong modification of the gray level (or color) values. 


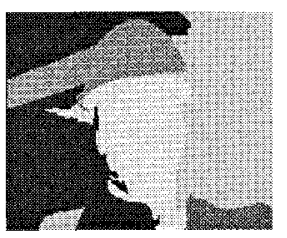

Level 5

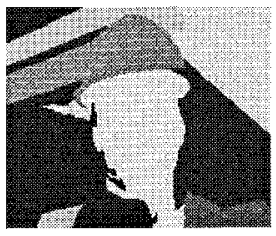

Level 4

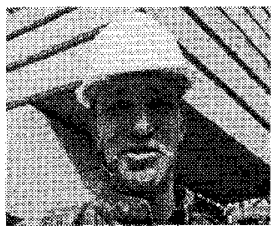

Original frame

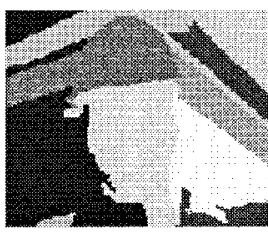

Previously coded frame

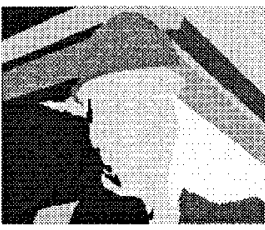

Projected partition

Partition Tree

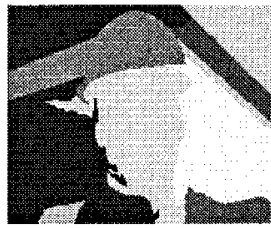

Final partition

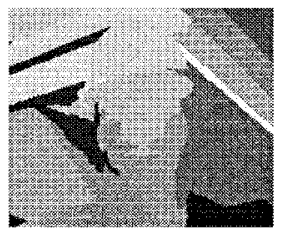

Level 2

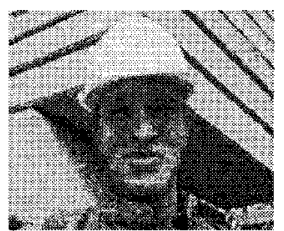

Coded frame

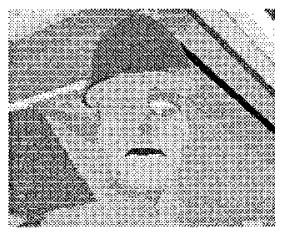

Level 1

Fig. 9. Example of interframe processing.
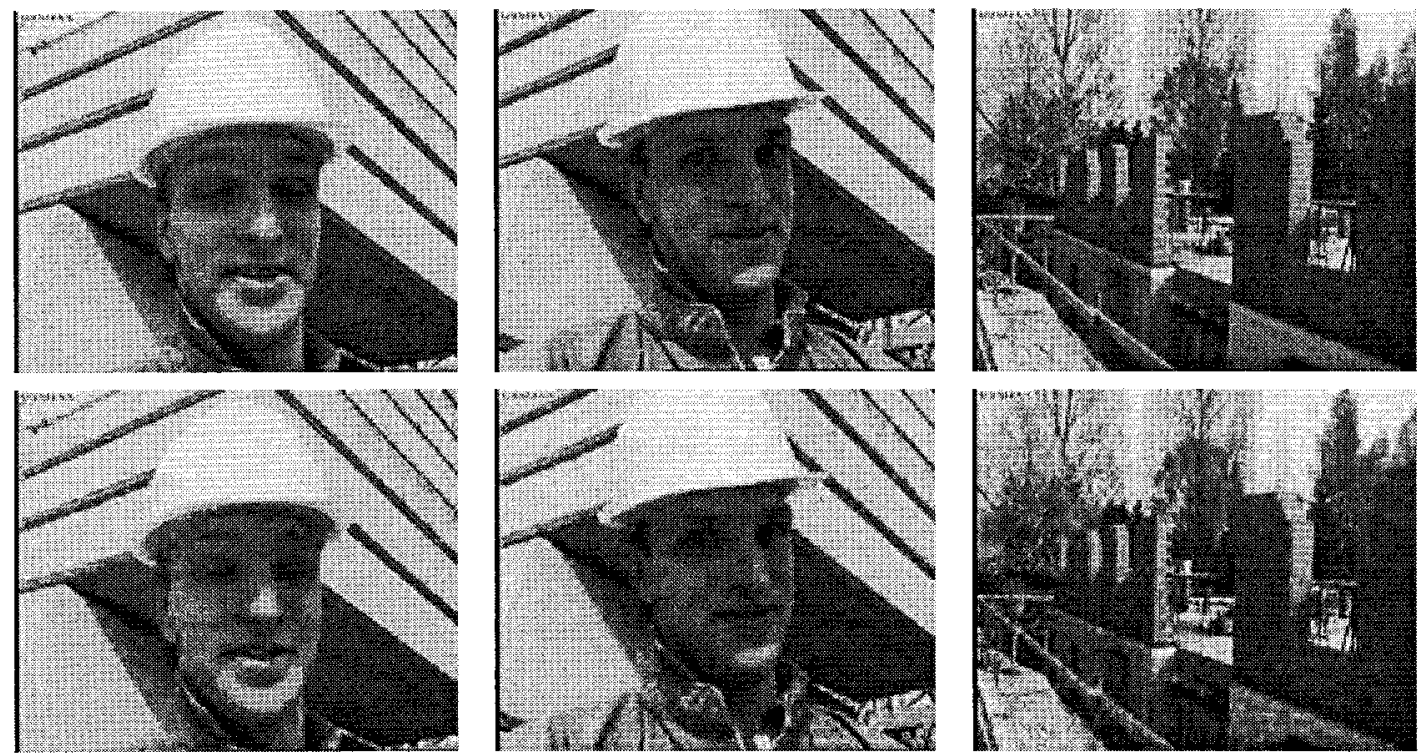

Fig. 10. Coding results for the Foreman sequence coded at $42 \mathrm{~Kb} / \mathrm{s}$ and 5 frames/s. First row: original frames 0 , 115 , and 235 . Second row: coded frames.

3) Code error contour: The actual coding of the error is done in two steps. The first step consists in coding the contours of the error regions. It relies on a differential coding of the contours. Indeed, the receiver already knows the contours that correspond to the compensated partition. Therefore, only the new contours have to be sent. Coding techniques similar to the one used for the intraframe mode can be used. The resulting partition can be seen as an overpartition because it involves contours of the compensated partition and of the segmentation.
The second step is to send the label of each region of the overpartition. First, the labels assigned on the receiver side are extracted. They correspond to the labels that were defined by the compensation process. Second, the most probable labels of each region are estimated. Note that, because of the simplification step, each region of the overpartition may involve several labels of partition to code. The most probable label is defined as the label that has the highest number of pixels in the considered region. The labels can be sent directly 


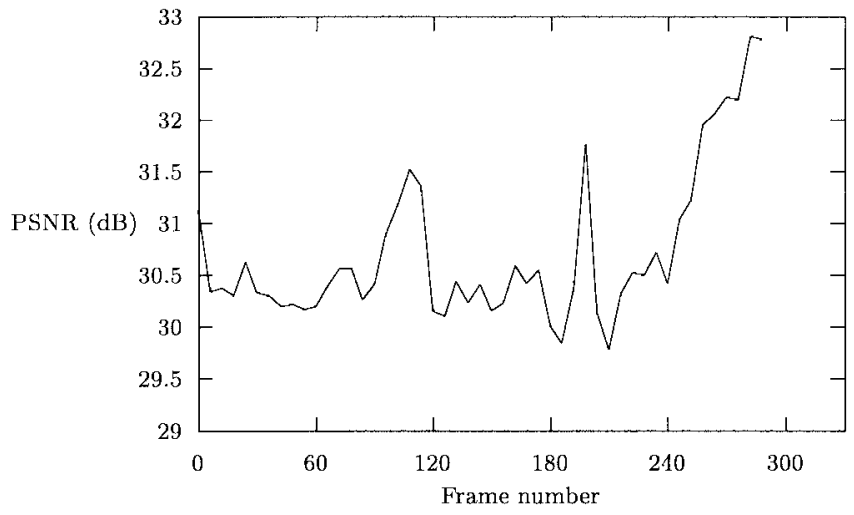

Fig. 11. Evolution of the PSNR for the Foreman sequence.

in the transmission channel but, in general, this would result in an excessive amount of information. To reduce this amount of information, specific coding strategies are used. Examples of these can be found in [28] and [29].

\section{F. Texture Compensation and Coding}

This section describes the different techniques that are used to actually code the texture of the image. Images are coded using the reconstructed partition and following the results of the Decision step. Therefore, interframe and intraframe mode region-based coding techniques are used. The main difference between these two modes is that, for interframe mode, a texture motion compensation is necessary.

1) Texture Motion Compensation: The aim of the motion compensation step is to build the best possible motion predicted image from the last coded image. In this coding approach, the motion compensation relies on the previous and current partitions as well as the motion information. These data are utilized to prevent the incorrect motion compensation of uncovered areas.

A restricted motion compensation is applied in order to predict the texture of a current region from that of a previous one. The restriction consists in compensating each pixel using the motion parameters of its region only if there is a correspondence between the label the prediction comes from and the current label. The texture of the areas that cannot be motion compensated due to this restriction is obtained by extrapolation of the compensated texture in a region-by-region basis [32]. This way, the texture of the uncovered areas that may appear in a given region is extrapolated only using texture information from this region.

The use of restricted motion compensation improves the quality of the compensated images. In addition to this improvement, this method has the very important feature of ensuring the region independence in the motion compensation step. This feature is necessary for content-based manipulations (such as drag and drop of objects), since it enables the separate decoding of any moving object.

2) Texture Coding: The goal of the region-based texture coding is to encode the texture and color information inside each region of an image partition. In intraframe mode, the

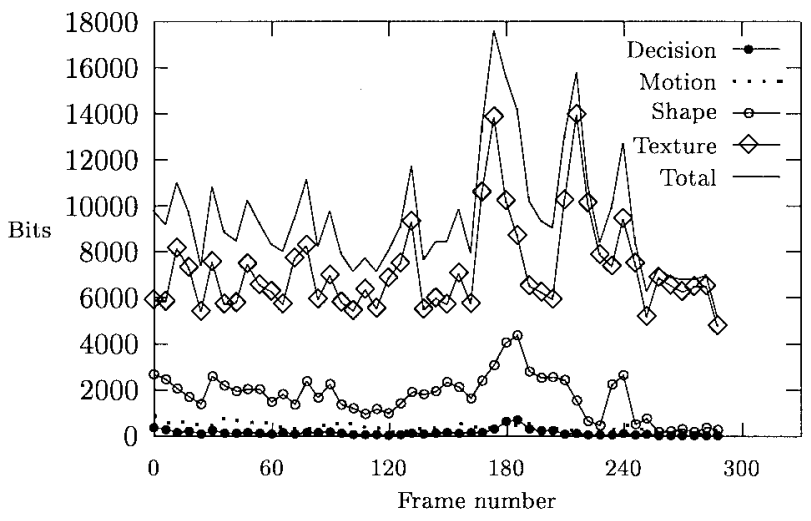

Fig. 12. Evolution of the number of bits per frame for the Foreman sequence.

texture is formed by the original pixel color values, whereas, in interframe mode, the texture to be coded is the motion compensation error. The algorithm flexibility allows the combination of several texture coding techniques. For each region, the technique giving the best compromise in the rate-distortion sense is selected. This selection is performed by the Decision step.

In particular, three different texture coding techniques have been used: polynomial approximation onto orthogonal bases [8], shape-adaptive discrete cosine transform (DCT) [35], [10], and region-based wavelets. For the first technique, cosine basis functions have been chosen as orthogonal bases given that they have proved to often outperform polynomial functions.

The basic idea of region-based wavelets has been introduced in [2]. Here, some improvements relying on the use of nonseparable bidimensional wavelet filters, combined with a region-based lattice vector quantization, are presented. The proposed approach is based on the use of the quincunx 2-D wavelet transform, which utilizes 2-D nonseparable low and high-pass filters [1].

When wavelets are applied on blocks or images, schemes based on 2-D nonseparable filters have proved to generally outperform those relying on separable filters. Thus, the idea is to generalize the use of 2-D nonseparable wavelet filters to region-based schemes. The process relies on two points:

1) The building of one low-pass and one high-pass segmentation mask at each level of resolution.

2) The efficient extension of the boundaries of the regions to minimize the reconstruction errors.

In order to build the low and high-pass subbands at each decomposition level while keeping a region-based approach, the segmentation mask (referred to as parent below) is split into two segmentation masks (children) corresponding to the low and high-pass subbands. The same process is performed for each region. To reconstruct the image at the synthesis side, the total number of pixels in a children region has to be equal to the number of pixels in the parent region. Moreover, as both square and quincunx grids are handled, according to the resolution level, the pixels of children regions are arranged in an efficient way in square and quincunx sampling grids. 

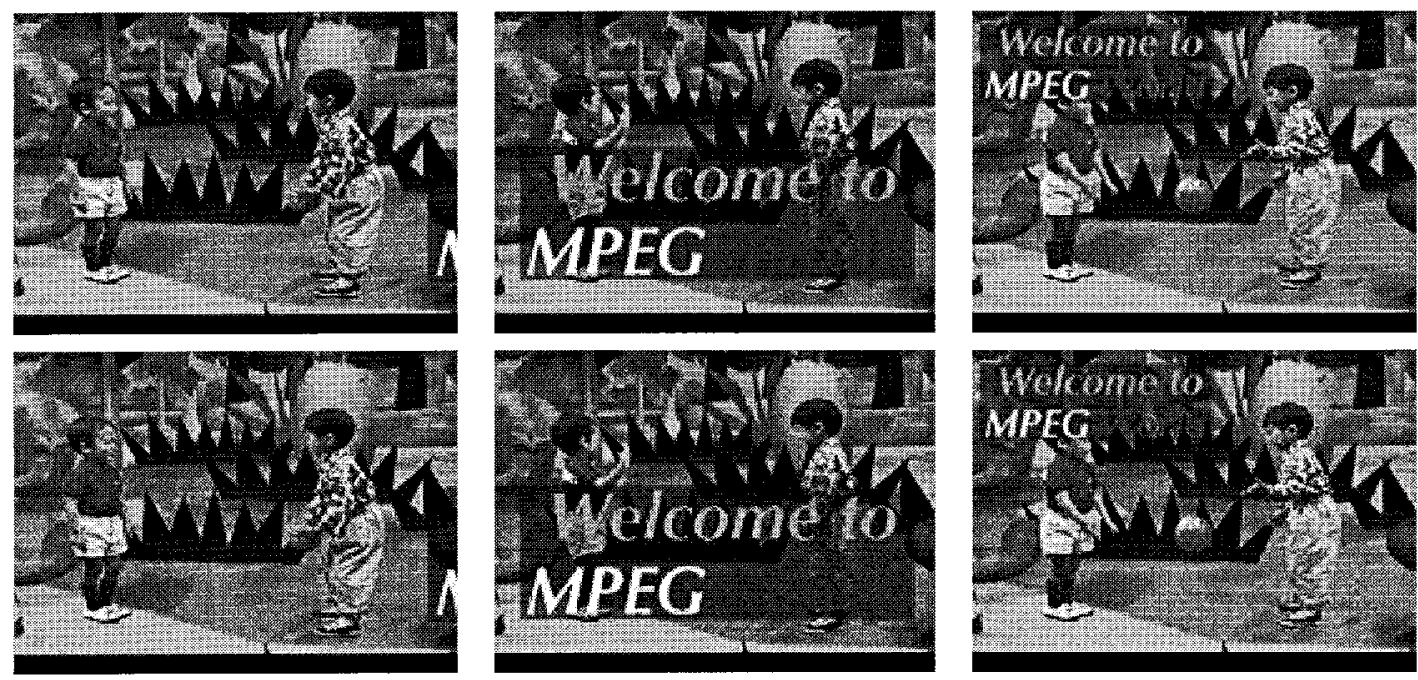

Fig. 13. Coding results for the Children sequence coded at $320 \mathrm{~Kb} / \mathrm{s}$ and 15 frames/s. First row: original frames 6, 100, and 192. Second row: coded frames.

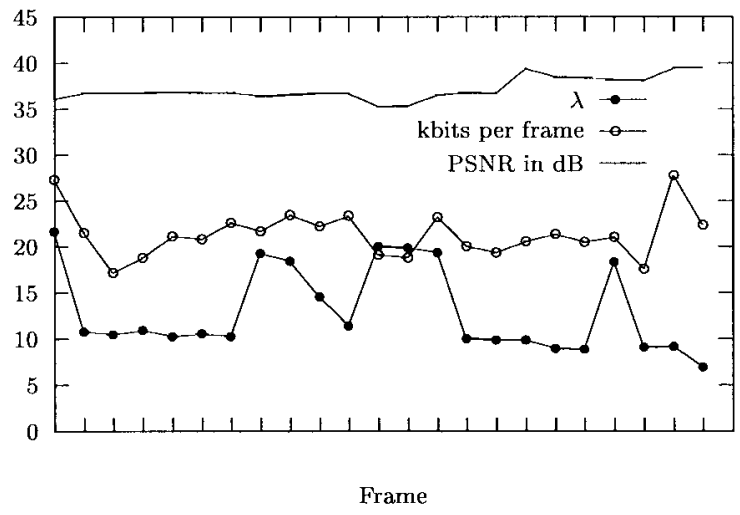

Fig. 14. Evolution of the PSNR, the number of bits, and the Lagrange parameter $\lambda$ of the Children sequence.

Once the splitting of the segmentation mask is achieved, the filtering and down-sampling of the image are performed independently on each region. To minimize the reconstruction errors on the region borders, an efficient extension of the boundaries is previously done. The method relies on the addition of $N$ layers around the region, where $N$ is the half size of the filter. The same process is iterated to build each layer. At each iteration, the magnitudes of the pixels of the extra layer are computed as the mean values of their neighboring pixels belonging to the region. The connectivity is four-neighbors whatever the grid. Regarding the low and high-pass filters used for the wavelet transform, bidimensional biorthogonal filters have been chosen. Filter coefficients are given in [1].

Once the wavelet coefficients have been computed, a modified lattice vector quantization process (LVQ) is performed to quantize them efficiently. In [5] a fast quantization algorithm for the LVQ based upon the lattice $D_{n}$ is presented for the case of block-based schemes. It groups the transformed coefficients into blocks and each block forms a vector that is quantized. This strategy takes into account the vertical and horizontal correlations of the transformed coefficients.
TABLE I

Bit Allocation of the Examples of Figs. 10 and 13

\begin{tabular}{||c|c|c|c|c|c||}
\hline \hline Sequence & Bit rate & Decision & Motion & Contour & Texture \\
\hline Foreman & $42 \mathrm{Kbits} / \mathrm{s}$ & $1.8 \%$ & $4.2 \%$ & $19.0 \%$ & $75.0 \%$ \\
\hline Children & $320 \mathrm{Kbits} / \mathrm{s}$ & $1.0 \%$ & $2.1 \%$ & $8.9 \%$ & $88.0 \%$ \\
\hline
\end{tabular}

In this work, this technique has been extended to the case of region-based schemes. First, the smallest rectangle of even horizontal and vertical length framing the current region is determined. This rectangle is split into blocks of size $2 \times 2$. For each block, the transformed coefficients belonging to the current region are stored in a vector to be quantized, whose size depends on the number of coefficients. This way, vectors of size 1,2, 3, or 4 are built. Therefore, according to the current vector size, an appropriate codebook has to be chosen for the quantization step.

\section{Performances in Coding Efficiency AND Content-BASEd Selective Coding}

The objective of this section is to show the capability of the algorithm to achieve good coding efficiency as well as to deal with content-based functionalities. In particular, the necessary modifications to allow the algorithm to carry out object tracking and content-based selective coding are summarized and some results presented.

\section{A. Coding Efficiency}

Let us start by an overview of the projection, the partition tree creation, the Decision process, and the coding. An example is given in Fig. 9. The image on the first row corresponds to the partition of the previous frame. This partition is projected and the projected partition can be seen in the center of the second row. This step defines the time evolution of the previously transmitted regions. Based on the projected partition, the partition tree is created: in the example of Fig. 9, levels 1 

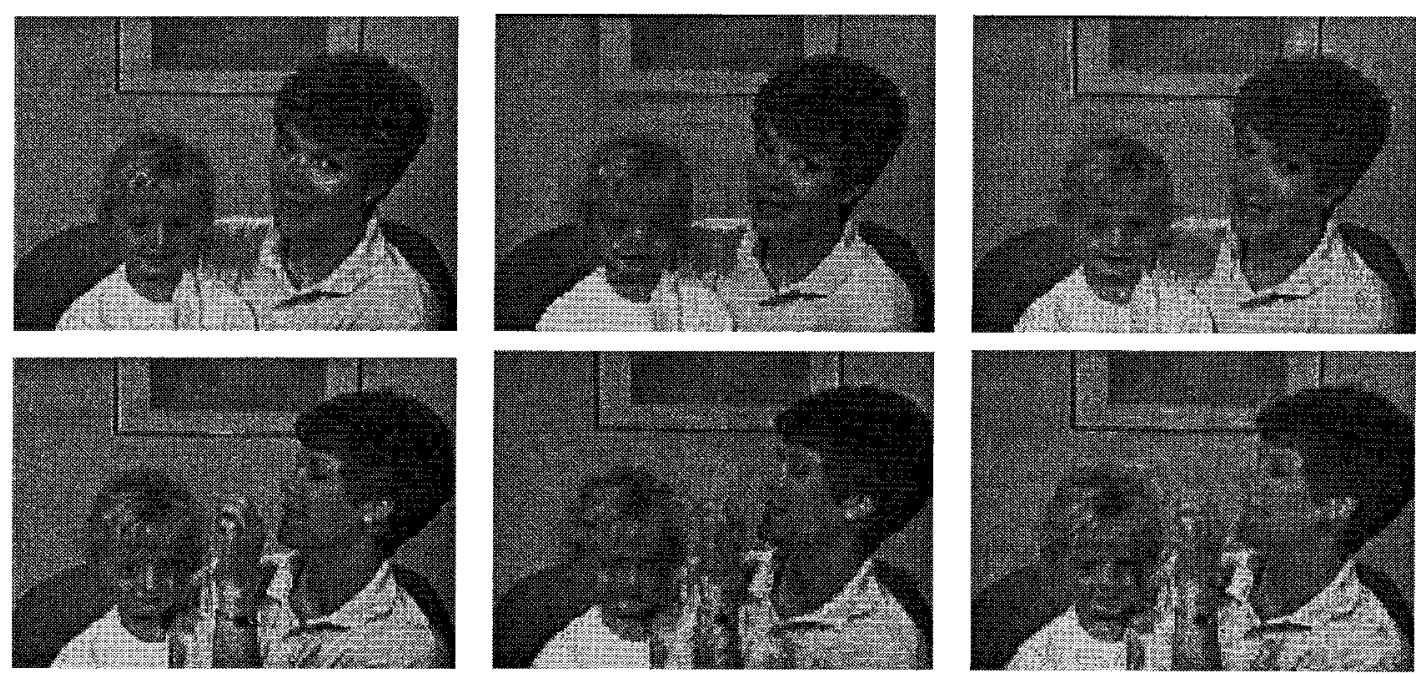

Fig. 15. Comparison between selective and nonselective coding of the head of the mother in frames 0 (first row) and 84 (second row) of the Mother and Daughter sequence. Left: original frames, Center: selective coding, Right: nonselective coding.

and 2 are obtained by hierarchical segmentation following a spatial homogeneity criterion. Note in particular how regions representing details of the face or of the background are introduced in the universe of regions. Levels 4 and 5 are created by merging regions with similar motion. Note here how background regions are merged because of their homogeneity in motion. The final partition is shown in the center of the lower row. In this partition, some regions are homogeneous in terms of gray level (regions corresponding to homogeneous part of the building) and others are homogeneous in motion (region corresponding to the face). Finally, the original as well as the resulting coded frames are shown in the lower row.

The algorithm has been mainly tested with QCIF and CIF sequences and bit rates from 24 to $320 \mathrm{~Kb} / \mathrm{s}$. In order to demonstrate the capability of the algorithm to cope with various types of sequences and scenarios, two different examples are presented in this section:

- The example of Fig. 10 shows the frames of the sequence Foreman coded at $42 \mathrm{~Kb} / \mathrm{s}$. This sequence is in QCIF format $(176 \times 144$ pixels $)$ and has been coded at 5 frames/s. The first row corresponds to the original frames 0,115 , and 235 and the second row to the coded frames. The time evolution of the peak signal-to-noise ratio (PSNR) and of the amount of bit per frame is shown in Figs. 11 and 12. The mean PSNR is around 30.5-31.0 dB and increases at the end of the sequence where the motion tends to disappear. In Fig. 12, together with the total number of bits per frame, the main pieces of information are shown: decision, motion, shape, and texture/color. It can be seen in particular that the bit-stream increases around frame 180 which is a difficult part of the sequence involving a strong panning. A significant part of the texture/color information cannot be compensated and has to be sent in intra mode. Moreover, a fairly high number of new regions are introduced in the partition. Finally, at the end of the sequence, the number of bits devoted to the shape information drops because the sequence becomes almost still and most regions are merged together.
- In turn, the example of Fig. 13 shows the frames of the sequence Children coded at $320 \mathrm{~Kb} / \mathrm{s}$. This sequence is in QCIF format and has been coded at 15 frames/s. The first row corresponds to the original frames 6, 100, and 192 and the second row to the coded frames. Details about the evolution of the PSNR and the number of bits per frame can be found in Fig. 14. Together with these results, we also show the time evolution of the Lagrange parameter $\lambda$ that defines the rate-distortion ratio.

The average (computed for the whole sequence) results of the bit allocation among the different types of information for both examples are summarized in Table I. Note that the Decision algorithm has selected a quite different strategy for the two bit rates: for low bit rates almost $20 \%$ of the bit stream is devoted to the partition information, whereas less than $10 \%$ is used for this type of information for higher bit rates. Finally, let us mention that by comparison with the algorithm presented in [32] using a much simpler bit allocation rule, the current algorithm provides between 2 and $3 \mathrm{~dB}$ of PSNR for very low bit rates $(30-40 \mathrm{~Kb})$.

\section{B. Content-Based Selective Coding}

In order to address content-based functionalities, images have to be described in terms of objects or groups of objects. These objects can be defined automatically or by the user, even on the receiver side in the case of an interactive application. Once they have been defined, the algorithm should make possible their tracking, within the coding scheme, through the time domain. Such a possibility opens, for instance, the ability to encode objects of interest with a higher quality than other parts of the image. In the proposed algorithm, the object tracking is mainly related to the Projection and partition tree blocks, whereas the selective coding is associated to the Decision block.

- Projection: The partition of the previous frame, which should already contain a region or set of regions describing the objects of interest, is projected following 
the double partition approach that has been presented in Section II-B. The position of the objects of interest in the current frame is obtained by regrouping the projection of the regions that formed the objects of interest in the previous frame.

- Partition Tree: The set of partitions that forms the partition tree is created having in mind the constraint introduced by the projection of the objects of interest. The different proposals of regions contained in the Partition Tree must not be in contradiction with the task of tracking the objects of interest. This translates into preventing, for partitions above the projected partition, the merging of regions with similar motion if they do not belong to the same object of interest. Such a merging would make impossible the separate tracking of the objects of interest.

- Decision: This block should yield a coding strategy leading to a lower distortion within the objects of interest than in other areas of the image. In order to obtain this selective coding, the bit allocation strategy used in the basic coding algorithm is slightly modified. In this case, if a target bit rate has to be reached, selective coding can be implemented by simply multiplying by a given factor the distortion in the regions forming the objects of interest.

- Coding: This block does not need to be changed since the coding techniques used in this functionality are the same as those used in the general case.

Fig. 15 presents the results of applying the previous algorithm to the sequence Mother and Daughter. The first column in Fig. 15 presents two original frames of the sequence, whereas the second and third columns show the decoded frames using selective and nonselective coding, respectively. The whole sequence has been coded in both cases at $30 \mathrm{~Kb} / \mathrm{s}$ and 5 frames/s. In this example, the head of the mother has been selected as the area of interest to be tracked and selectively coded. The selective coding has been carried out by multiplying by a factor ten the distortion inside this area of interest. The different qualities obtained for the heads of both persons in the scene should be highlighted. In addition, note that the evolution of the mother's face is correctly tracked.

\section{CONCLusions}

This paper has presented and discussed a generic video coding algorithm. It is a region-based scheme where regions are tracked in time. The bit allocation plays a central role in the coding process, it makes the link between the analysis and the synthesis parts of the encoder. While defining the coding strategy, it leads to the optimization of the partition as well as of the set of coding techniques to be used in each region.

One of the advantages of the approach is that the basic scheme which has been developed to achieve compression can be easily modified to deal with a large set of content-based functionalities. Moreover, the structure itself of the algorithm allows the integration and the comparison of new tools such as motion estimation, contour coding, texture coding, projection, etc.
The computational complexity of the encoding process is largely dominated by the number of different texture coding techniques that are proposed to the Decision. In the description of the algorithm, we have implicitly assumed that all techniques were tested for all regions. This is of course not necessary and, in practice, one can design rules to $a$ priori know that it is useless to try one technique in a given region.

The proposed algorithm can be improved in several directions. Beside improving each processing block, there are two particular points of interest. First, the distortion measure that has been used is the squared error because it is a simple additive criterion. However, it could be replaced by a more perceptually relevant criterion. Second, the optimization has been carried on a frame basis. Improvements can certainly be achieved if the optimization is done on a larger time scale.

\section{REFERENCES}

[1] M. Barlaud, P. Solé, T. Gaidon, M. Antonini, and P. Mathieu, "Pyramidal lattice vector quantization for multiscale image coding," IEEE Trans. Image Processing, vol. 3, no. 4, pp. 367-381, July 1994.

[2] H. J. Barnard. "Image and video coding using a wavelet decomposition," Ph.D. dissertation, Delft University, 1994

[3] J. Benois, L. Wu, and D. Barba, "Joint contour-based and motion-based image sequences segmentation for TV image coding at low bit rate," in Visual Communication and Image Processing. Chicago: SPIE, Sept. 1994, pp. 1074-1085.

[4] P. Bouthemy and E. François, "Motion segmentation and qualitative dynamic scene analysis from an image sequence," Int. J. Computer Vision, vol. 10, no. 2, pp. 157-182, 1993.

[5] J. H. Conway and N. J. A. Sloane, "Fast quantizing and decoding algorithms for lattice quantizers and codes," IEEE Trans. Inform. Theory, vol. 28, no. 2, pp. 713-718, Mar. 1982

[6] I. Corset, L. Bouchard, S. Jeannin, P. Salembier, F. Marqués, M. Pardàs, R. Morros, F. Meyer, and B. Marcotegui, "Segmentation-based coding system allowing the manipulation of objects (SESAME)," Tech. Rep., ISO/IECJTC1/SC29/WG11/MPEG95/408, LEP, UPC, CMM, Nov. 1995.

[7] J. L. Dugelay and H. Sanson, "Differential methods for the identification of 2D and 3D motion models in image sequences," Signal Processing. Image Communication, vol. 7, pp. 105-127, 1995.

[8] M. Gilge, T. Engelhardt, and R. Mehlan, "Coding of arbitrarily shaped image segments based on a generalized orthogonal transform," EURASIP, Image Communications, vol. 1, no. 2, pp. 153-180, Oct. 1989.

[9] C. Gu and M. Kunt, "Very low bit-rate video coding using multi-criterion segmentation," in First IEEE Int. Conf. Image Processing, Texas, USA, Nov. 1994, vol. II, pp. 418-422.

[10] E. Jensen, K. Rijkse, I. Lagendijk, and P. van Beek, "Coding of arbitrarily shaped image segments," presented at Workshop on Image Analysis and Synthesis in Image Coding, Berlin, Germany, Oct. 1994.

[11] M. Kunt, A. Ikonomopoulos, and M. Kocher, "Second generation image coding techniques," Proc. IEEE, vol. 73, no. 4, pp. 549-575, Apr. 1985.

[12] F. Marqués, "Motion stability in image sequence segmentation using the watershed algorithm," in Third Workshop on Mathematical Morphology and its Applications to Image Processing, P. Maragos, R. W. Schafer, and M. A. Butt, Eds., Atlanta, USA: Kluwer, May 1996, pp. 321-328.

[13] F. Marqués, B. Marcotegui, and F. Meyer, "Tracking areas of interest for content-based functionalities in segmentation-based video coding," in IEEE Int. Conf. Acoustics, Speech, Signal Processing, ICASSP'96, Atlanta, USA, May 1996, pp. 1224-1227.

[14] F. Marqués, J. Sauleda, and A. Gasull, "Shape and location coding for contour images," in Picture Coding Symp., Lausanne, Switzerland, pp. 18.6.1-18.6.2, Mar. 1993.

[15] F. Marqués, V. Vera, and A. Gasull, "A hierarchical image sequence model for segmentation: Application to object-based sequence coding," in Proc. SPIE Visual Communication and Signal Processing-94 Conference, VCIP'94, Chicago, USA, Oct. 1994, pp. 554-563. 
[16] F. Meyer and S. Beucher, "Morphological segmentation," J. Visual Commun. Image Representation, vol. 1, no. 1, pp. 21-46, Sept. 1990.

[17] R. Morros, F. Marqués, M. Pardás, and P. Salembier, "Video sequence segmentation based on rate-distortion theory," in SPIE Visual Communication and Image Processing, VCIP'96, vol. 2727, Orlando FL, USA, Mar. 1996, pp. 1185-1196.

[18] MPEG-4 Proposal Package Description (PPD). Technical Report ISO/IEC JTC1/SC29/WG11, MPEG, July 1995.

[19] H. G. Musmann, M. Hötter, and J. Ostermann, "Object-oriented analysis-synthesis coding of moving images," Signal Processing, Image Commun., vol. 1, no. 2, pp. 117-138, Oct. 1989.

[20] A. Ortega, K. Ramchandran, and M. Vetterli, "Optimal bufferconstrained source quantization and fast approximations," in Proc. IEEE Int. Symp. Circuits Systems, vol. 1, May 1992, pp. 192-195.

[21] M. Pardàs and P. Salembier, "Joint region and motion estimation with morphological tools," in Second Workshop on Mathematical Morphology and its Applications to Signal Processing, J. Serra and P. Soille, Eds. Fontainebleau, France: Kluwer Academic Press, Sept. 1994, pp. 93-100.

[22] _ "Time-recursive segmentation of image sequences," in $E U$ SIPCO 94, VII European Signal Processing Conference, Edinburgh, U.K., Sept. 13-16, 1994, pp. 18-21.

[23] M. Pardàs, P. Salembier, and B. González, "Motion and region overlapping estimation for segmentation-based video coding," in IEEE Int. Conf. Image Processing, ICIP'94, vol. II, Austin, TX, Nov. 1994, pp. 428-432.

[24] M. Pardàs, P. Salembier, F. Marqués, and R. Morros, "Partition tree for segmentation-based video coding," in IEEE Int. Conf. Acoustics, Speech, Signal Processing, ICASSP'96, vol. IV, Atlanta, USA, May 1996, pp. 1983-1986.

[25] K. Ramchandran and M. Vetterli, "Best wavelet packet bases in a ratedistortion sense," IEEE Trans. Image Processing, vol. 2, no. 2, pp. 160-175, Apr. 1993.

[26] E. Reusens, "Joint optimization of representation model and frame segmentation for generic video compression," EURASIP Signal Processing, vol. 46, no. 11, pp. 105-117, Sept. 1995.

[27] P. Salembier, "Morphological multiscale segmentation for image coding," EURASIP Signal Processing, vol. 38, no. 3, pp. 359-386, Sept. 1994.

[28] _ _ "Motion compensated partition coding," in SPIE Visual Communication and Image Processing, VCIP'96, vol. 2727, Orlando, FL, Mar. 1996, pp. 403-415.

[29] P. Salembier, F. Marqués, and A. Gasull, "Coding of partition sequences," in Video Coding: The Second Generation Approach, L. Torres and M. Kunt, Eds. Kluwer, 1996.

[30] P. Salembier and M. Pardàs, "Hierarchical morphological segmentation for image sequence coding," IEEE Trans. Image Processing, vol. 3, no. 5, pp. 639-651, Sept. 1994.

[31] P. Salembier and J. Serra, "Flat zones filtering, connected operators and filters by reconstruction," IEEE Trans. Image Processing, vol. 3, no. 8, pp. 1153-1160, Aug. 1995.

[32] P. Salembier, L. Torres, F. Meyer, and C. Gu, "Region-based video coding using mathematical morphology," Proc. IEEE (Invited paper), vol. 83 , no. 6 , pp. 843-857, June 1995 .

[33] H. Sanson, "Toward a robust parametric identification of motion on regions of arbitrary shape by nonlinear optimization," in Proc. IEEE Int. Conf. Image Processing, ICIP'95, vol. I, Oct. 1995, pp. 203-206.

[34] Y. Shoham and A. Gersho, "Efficient bit allocation for an arbitrary set of quantizers," IEEE Trans. Acoustics, Speech, Signal Processing, vol. 36, pp. 1445-1453, Sept. 1988.

[35] T. Sikora, S. Bauer, and B. Makai, "Efficiency of shape-adaptive 2D transforms for coding of arbitrarily shaped image segments," IEEE Trans. Circuits Syst. Video Technol., vol. 5, no. 1, pp. 59-62, Feb. 1995.

[36] G. Tziritas and C. Labit, Motion Analysis for Image Sequence Coding, vol. 4 of Advances in Image Communication. Elsevier, 1994.

[37] P. Willemin, T. Reed, and M. Kunt, "Image sequence coding by split and merge," IEEE Trans. Commun., vol. 39, no. 12, pp. 1845-1855, Dec. 1991.

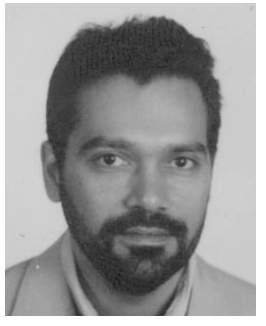

Philippe Salembier received a degree from the Ecole Polytechnique, Paris, France, in 1983 and a degree from the Ecole Nationale Supérieure des Télécommunications, Paris, France, in 1985. He received the Ph.D. degree from the Swiss Federal Institute of Technology (EPFL) in 1991. He was a Postdoctoral Fellow at the Harvard Robotics Laboratory, Cambridge, MA, in 1991.

From 1985 to 1989 he worked at Laboratoires d'Electronique Philips, Limeil-Brévannes, France, in the fields of digital communications and signal processing for HDTV. In 1989, he joined the Swiss Federal Institute of Technology in Lausanne, Switzerland, to work on image processing. At the end of 1991, after a stay at the Harvard Robotics Laboratory, he joined the Polytechnic University of Catalonia, Barcelona, Spain, where he teaches digital signal and image processing. His current research interests include image and sequence coding, image modeling, segmentation problems, texture analysis, mathematical morphology, and nonlinear filtering.

$\mathrm{He}$ is currently serving as an Area Editor of the Journal of Visual Communication and Image Representation (Academic Press) and as an AdCom officer of the European Association for Signal Processing (EURASIP) in charge of the edition of the Newsletter.

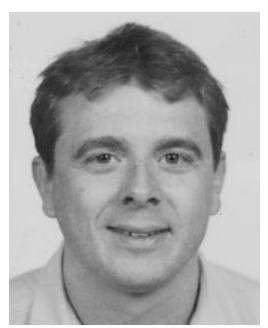

Ferran Marqués graduated from the Polytechnic University of Catalonia (Barcelona) in December 1988. He received the Ph.D. degree from the Polytechnic University of Catalonia (UPC) in December 1992.

From 1989 to June 1990 he worked in the Swiss Federal Institute of Technology in Lausanne (EPFL) in the group of Digital Image Sequence Processing and Coding. In June 1990, he joined the Department of Signal Theory and Communications of the Polytechnic University of Catalonia (UPC). From June 1991 to September 1991, he was with the Signal and Image Processing Institute at the University of Southern California in Los Angeles, CA. $\mathrm{He}$ is now an Associate Professor at UPC teaching Image processing and Communication. His main areas of interest are still image and sequence segmentation, still image and sequence coding, motion estimation and compensation, mathematical morphology and biomedical applications.

Dr. Marqués obtained the Spanish Best Ph.D. Thesis on Electrical Engineering Award-1992.

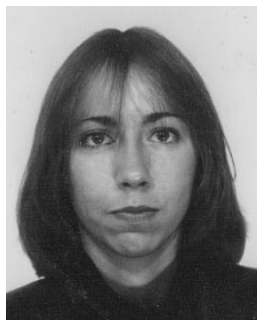

Montse Pardàs received the M.S. degree in telecommunications and the Ph.D. degree from the Polytechnic University of Catalonia, Barcelona Spain, in 1990 and 1995, respectively.

Her main research activity deals with nonlinear signal processing with a special emphasis on mathematical morphology. She is particularly interested in analysis problems for sequences such as segmentation, motion estimation, and coding.

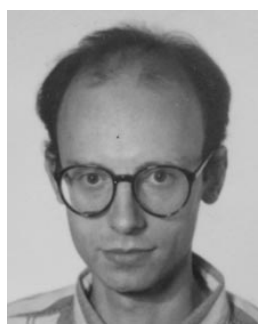

Josep Ramon Morros received the M.S. degree in physics in 1989 from University of Barcelona, Spain. He is currently working toward the Ph.D. degree in the Signal Processing Department of the Polytechnic University of Catalonia, Barcelona, Spain.

His current research interests include image and sequence coding, segmentation, and mathematical morphology. 


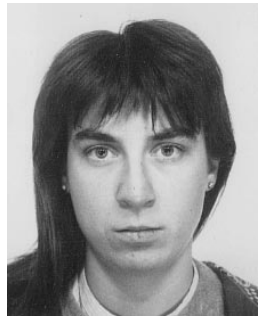

Isabelle Corset was born in France in 1969. She graduated from Ecole Nationale Supérieure des Télécommunications (ENST), Paris, France, in 1991.

Since 1991, she has been a research engineer at Laboratoires d'Electronique Philips SAS (LEP), Limeil-Brévannes, France, in the Image and Communication group. Her main research activity deals with image analysis and image coding. She was involved in MPEG-2 video development and was active in the RACE-MAVT project. She is now involved in the video aspects of MPEG-4.

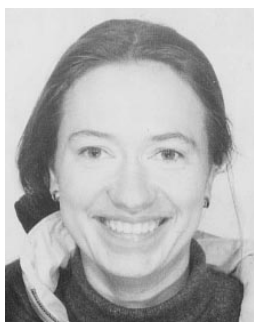

Sylvie Jeannin was born in France in 1969. She graduated from Ecole Nationale Supérieure des Télécommunications (ENST), Paris, France, in 1992.

Since 1993, she has been a research engineer at Laboratoires d'Electronique Philips SAS (LEP), Limeil-Brévannes, France, in the Image and Communication group. Her main research activity deals with image analysis and image coding, with a special emphasis on motion estimation related problems. She is involved in COST 211ter group and in the ACTS-MoMuSys project and works on the MPEG-4 video coding methods.

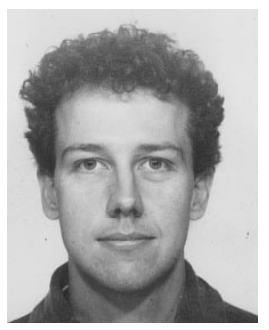

Lionel Bouchard was born in France in 1968. He graduated from Ecole Nationale Supérieure des Télécommunications (ENST), Paris, France, in 1992.

Since 1993, he has been a research engineer at Laboratoires d'Electronique Philips SAS (LEP), Limeil-Brévannes, France, in the Image and Communication group. His research interests include image analysis and coding as well as new languages (Java, VRML). He was active in the RACE-MORPHECO project. He is now involved in the systems aspects of MPEG-4, the future standard for composition and coding of multimedia objects.

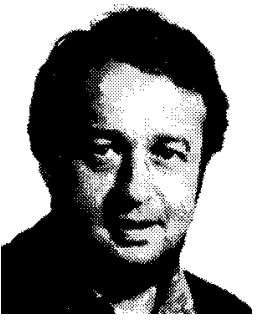

Fernand Meyer was born in 1952. He received the engineering degree and the Ph.D. degree from the Ecole des Mines de Paris in 1975 and 1979, respectively.

He has been with the Centre de Morphologie Mathématique (CMM) since 1975, where he is now in charge of the coding activities and of the applications of image processing to medical imaging. His research interests are in skeleton, morphological segmentation, algorithmic design, and 3-D mathematical morphology.

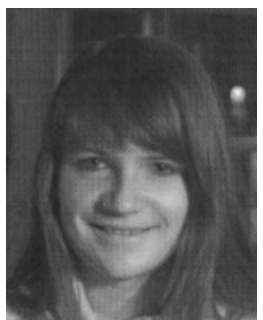

Beatriz Marcotegui was born in Pamplona, Spain, in 1968. She received the M.S. degree in telecommunications engineering in 1992 from the Polytechnic University of Catalonia, Barcelona, Spain. She received the Ph.D. degree from the School of Mines of Paris, Fontainebleau, April 1996.

Her research interests include digital image processing and mathematical morphology. Within these areas she has been particularly involved in image sequence segmentation and video coding. 